\title{
XXXIII.
}

\section{Die Dioskuren.}

Castor und Pollux erscheinen in antiker tradition melirfach als übermenschliche mitstreiter und helfer zum siege; zugleich dann als gute boten, die mit märchenhafter schnelle den sieg nach hause melden. In der schlacht am see Regillus ${ }^{1}$ ) waren sie an der spitze der römischen reiter, grösser und schöner als jetzt sterbliche menschen sind ${ }^{2}$, , hoch zu ross aber fast noch knaben. Desselben tages, in später stunde, erschienen sie in Rom, erzählend von dem römischen siege und der flucht der Latiner; in den zügen der jünglinge las man noch die wuth des streites; sie wuschen sich am quell nahe dem Vestatempel, ihre pferde trieften von schweiss. Und in den abhängen des Albanerberges zeigte man im harten gestein ${ }^{5}$ ) die spur eines eingedrückten hufes, nicht eines irdischen hufes. So konnte nur der Xanthos (der gelbe) oder der Kyllaros (celes, $\left.x \varepsilon^{\prime} \eta \eta \varsigma\right)$ gestampft haben oder welches andere von ihren rossen ${ }^{4}$ ) die Zeusknaben gerade ritten. Die Regillusschlacht fand idihus Quinctilibus a. u. 258 Varr. $=$ a. Ch. 496 (Dionys. Bal., Fasti.) statt, d. h. an einem vollmondstage ${ }^{5}$ ) in der mittsommerzeit; als variante für das anno hat man nach Liv. II, 21 a. u. $255 \mathrm{Varr} .=$ a. Ch.

1) Cic. Nat. Deor. II, 2, 6 .

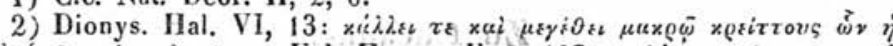

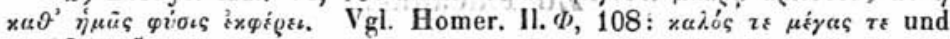
$Y, 287$ : ỡo vĩy B poroi ziouv.

3) In silice Cic. N. D. III, 5 \$. 12.

4) Stesichorus fragm. 1 nennt vier, zunächst wohl aber für den wagen; als muster für das wagenrennen in Olympia.

5) Von den idib. Maiis der Decemvirn a. Ch. 450 redend sagt Dio-

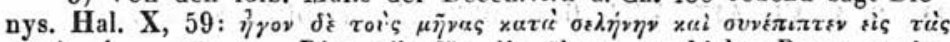

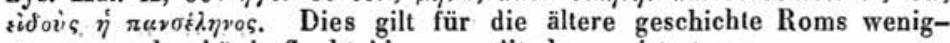
stens von der königsflucht bis zur solitudo magistratuum. 
499. Ignorirt man die letztere und wählt a. u. 258 Varr., so wird, wenn man das, erste jahr der freiheit vom frühling 510 a. Ch. begonnen hat, bis dahin 509 reichend, auch das jahr der Regillusschlacht mit dem frühling 497 a. Ch. beginnen und id. Quinctil. auf den vollmond des Skirophorion, altmetonisch oder neumetonisch, auskommen. Dieser vollmond aber ist wie der des dies Cremerensis nahezu solstitial, indem die beiden gemeinten jahre homologe der Enneakaidekaeteris sind.

Wofern also die römische tradition mit ihrer datirung den mittsommertag, den längsten des jahres bezielte, so kann man auch unseren volksglauben zum vergleich anführen; wer kennt nicht die St. Johannisnacht und ihre wunder? Wie das wintersolstiz auf den heiland, so wurde das des sommers auf Johannes gedeutet 6 ); lange indess ehe der tag den namen Johannes Baptista empfing, war er bei classischen ${ }^{7}$ ) wie bei germanischen heiden ein hoher und hochgefeierter. Zufolge der nativität des Romulus, welche sich Varro vom Tarutius erbat (röm. daten p. 8), kommt die empfängniss des Marssohns auf die sommer. wende und das Festum Quirini a. d. IV K. Julias oder 28 juni kann schwerlich etwas anderes bedeuten, ungeachtet zu Caesars zeit allerdings die wende nicht am 28,juni statt fand. Bei den alten Germanen ward der tag als eine hohe freudenzeit began. gen (johannisfeuer: s. Grimm mythol. p. 589); aberglaube schloss sich an, wie nur in der johannisnacht der farrensame zu finden ist, vermöge dessen man an der kirchthür sitzend alle todten des jahres sieht und was dergleichen mehr ist. Die zeit des höchsten sonnenstandes', gleichsam der jahresmittag ist etwas uatürlich gegebenes, eine halkyonische jahresstille, während welcher die einbildungskraft ${ }^{8}$ ) ungehemmter schaffen mag. Allein es gilt noch andere beispiele der kastorenepiphanie zu prüfen.

Vatinius ein römischer landmann reiste von Reate nach Rom und befand sich nachts auf der salarischen strasse (Cic. N. D. III, 5 §. 11). Da erschienen ihm zwei jünglinge auf

6) Weidenbach Calendariun p. 201.

7) Nur dass die lunarische zeitrechnung das solstiz an eine phase zu knüpfen strebte; bei den kaum merklich verschiedenen taglängen der mittsommerzeit konnte sich der cultus und der aberglaube einen tag aussuchen, auf den der vollmond kam.

8) Auch im winter während der tage um die wende besinnt sich Zeus, $\pi \iota v \dot{\sigma} \sigma \varepsilon \iota$ : s. Simonidis fr. 14 Bergk. 
weissen rossen, sie meldeten die besiegung ${ }^{9}$ ) des Perseus durch Aemilius Paullus, welcher am 22. juni jul. a. Ch. 168 bei Pydna stattgefunden hatte. Das datum ist gesichert durch die mondfinsterniss am abend vorher (Ideler II, p. 104). Rechnet man nun den 22. juni, den tag von Pydna, als den ersten, so ist der vierte: 25 juni julianisch. Die zeit des solstizes aber kommt für dies jahr ungefälır auf morgens 5 uhr 26 juni; folglich beginnt der heilige abend des mittsommertages schon mit dunkelwerden am 25. juni. Vatinius also, wenn er am 25. juni vom einbruche der nacht übereilt wird, reiset in den johannisabend hinein und die johannisnacht bringt auch hier die epiphanie der Zeusknaben. Das wunder nun bestand darin, dass am tage da Paullus seine briefboten absandte ${ }^{10}$ ) die kunde schon in Italien war. So schnell konnten nur die himmlischen couriere reiten, die irdischen kamen erst a. d. VI K. Oct. um die zweite römische stunde in die stadt, morgens 13. juli jul. a. Ch. 168, ein und zwanzig tage nach der schlacht, aclitzehn nach ihrer entsendung.

Drittens endlich, als am flusse Sagra die italischen Lokrer und Rheginer 10,000 mann stark gegen 130,000 Krotoniaten einen bis zum unglaublichen glorreichen sieg gewannen, wurde die siegesbotschaft noch an demselben tage zu Olympia vernommen, wo man eben die olympischen spiele feierte. Die altäre,

9) Liv. 45, 1: pugnatum in Macedonia et devictum regem esse. Nach Cic. N. D. II, 2: regem Persen illo die (25. juni) captum, was falsch ist so wie Lactant. II, 7, der das captum des Cicero mit dem devictum des Livius zusammenflickt: nuntiantes eo die (25. juni) regem Persem victum atque captum. Die persönliche gefangennehmung des macedonischen königs fand nicht wenige tage später statt. Wenn Cicero von einer dem schreiben des Paullus zufolge nachgehends constatirten übereinstimmung des datums spricht - a Paullo litteris allatis quum idem dies constitisset - so kann er in seiner quelle weiter nichts gefunden haben als dass das schreiben des Paullus datirt war von dem tage an welchem Vatinius die Dioskurenmeldung empfangen hatte. Denn auf Livius darstellung 44,45 passt der 25 juni für die absendung des briefes. (Bei der tagzählung wird es wohl das richtigste sein den terminus a quo milzurechnen).

10) Liv. 44, 45: Tertio die Perseus quam pugnatum erat, Amphipolin venit. Inde oratores cum caduceo ad Paullum misit. Diese gesandten konnten etwa quinto (sexto) die post pugnam bei dem Paullus sein; das folgende "mittlerweile" (interim) wird hiernach vor die ankunft der caduceatoren kommen: interim .... principes amicorum regis .... ipsi ad consulem profecti Romanis se dedunt; hoc idem et alii deinceps melu perculsi parabant facere. Consul nuntiis victoriae... cum litteris Romam missis cet. Diese boten werden also quarto post die quam cum rege est pugnatum $(45,1)$ abgegangen sein. 


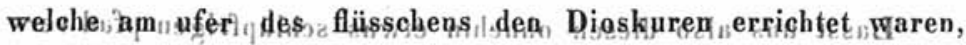
zeigten noch dep ispäten nachwelt, wer es gewesen, dem die, Lo-

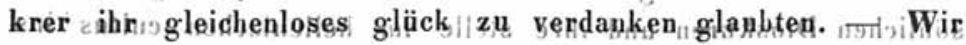
kennen das jahn der, schlacht nicht, 11 ) doch zeigt die erwäls;

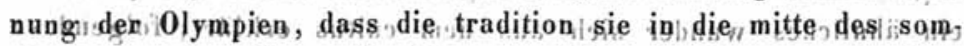
mers iverlegte. Denn die feier der olympien kommt auf eqinę an das solstiz sich lehnenden vollmond, Wie nahe ider mit, jenen

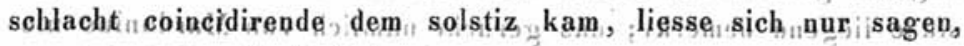
wenn $\backslash$ das $\mid$ jahr äberliefert wäre,

noimWird (uns, um diesse beziehung der Kastoren anf i den sommer zulerklären, vielleicht die nepere mythenforschung eipige hälfe gewähren $\}_{3}$ sie findet in den Kastoren erste lichthlicke der morgensonne, womit vieles recht hübsch stimmt. ${ }_{19}$ Wenn ihre

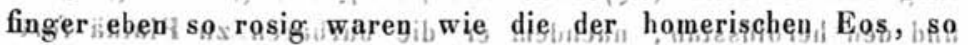
sieht $_{11}$ man $_{n}$ wohl wie jenem ahnherrn der Domitier, als die finger des zeusknaben seinen bart berührten, das schwarze haar in

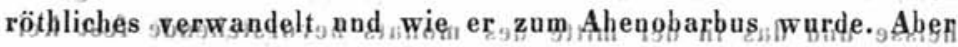
weit mehr $_{\text {nnerledigte }}$ fragen und zweifel bleiben übrig wilo Wenn Castor und Pollux die das dupkel durchbrechepden strahlen des frühliebtes anzeigen siweshalb sind $_{11}$ es, dann eben zwei ? ist der

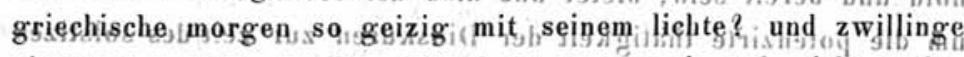
nimmt man doch so als wären es nur zwei und nicht mehr, gleichsam nothwendig und abgeschlossen in ihrer zweibeit t] Die verfrühungen der sonnenaufgänge allein ergeben noch das solstiz njęht, „welches vielmẹr/statt hat wenn die wiederum eintretende verspätung des, sonnenaufgangs nahe bevorsteht, der schon eine

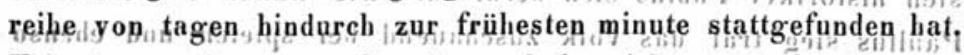
Ueberhaupt ging man für das solstiz nicht yom morgen aus sondern vom mittag und dem mittagsschatten, Wer die Kasto. ren, für erste I morgenstrahlen erklärt, wird zugeben müssen, dass

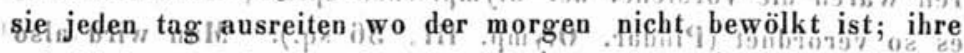
solstitialen epiphanien lassén sich so nicht füglich erklären, woraus indess nicht folgt, dsss die mythologen in jeder beziebung falsch gedentet haben.

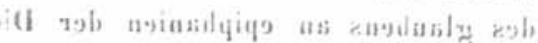

-9tili) Niebuhr setzt die sagraschlacht jedenfalls richtig hach" der zer h störung son: Sybarisı Nur traut mah sich liaum dièse letztére mit, sicherheit auf $510 \mathrm{~g}$. Ch 20 bringen nach Diodor; es ist die epoche des Timaus, doch im ungefahren kann ja die bestimmung richtig séin. Die

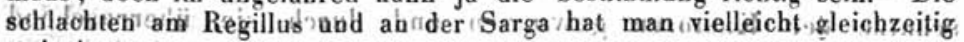
gedaçbt

Philologas. 195:1 XI. Jahrg. 4

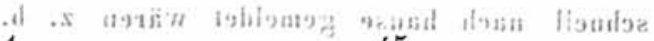
* Brought to you by | New Y of 5 University 


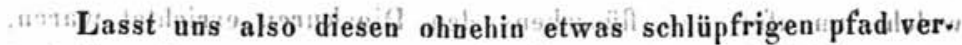
lassen und statt dér grundidée lieber' ilire verkörperung, die persönlichen Dioskuren "und ilire stelle im hellenischen cultus ins auge ${ }^{7}$ fassen. Hieriñ bestärkt sogleich lin anderes beispiel, wo wo eì äbnlichè"'t" wunder sich aus dem cultus erklärt; Ofolgendes : als bei Mycale gegen den barbaren vorgegangen ward, durch. flog ein gerücht die reihen und ein heroldsstab ward am ge: stade liëgend bemerkt; das gerücht nämlich von_Mardonius bie. derlage bei Platää, welche morgens'stattgefunden, wắlırend man bei "Mycale" abends focht. "Die schlacht fand am 3. Boedromion statt, also im monate der Eleusinien. Dié eleusimischen mächte schieiret das gegen die "barbareh gerichfete gebot der fernhal-

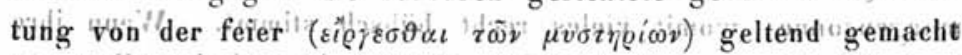
und dén heroldsstab, nachdem er "die barbaren zu Platä fortgewiesên, za den gléchen zwecke nach Myeale gesandt 'zut lia ben, damit auch hier der stumine bote die barbaren fortgehen heisse und das in der mitte des monats bevorstehende fest l kei" nen "mehr zur stelle finde, der die eleusinischen sutzungen beleidigt hätte. Eing jede gottheit wird an ihreñ feste besonder's hold und bereit sein ; bietet uns also der festkaleirder etwas dar um die potenzirte thátigkeit der 'Dioskuren' zur' zeit'des'solstizes zu erklären? Der sieger" am see "Régillús gelobte den hìmmlíschen hel fern auser eivem tempel auch spiêle "und" das "sind"die", welche

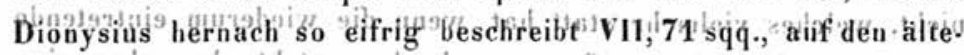

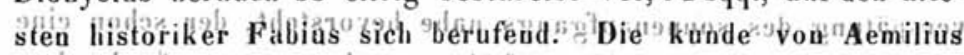
Paullus sieg traf das volk" zuschauerd "bei spielen und ébenso traf die Sagraschlacht "huf die Olyinpient. Dém Castoriund ${ }^{2} \mathbf{P}$ il. lux galt das fest welches Postumitus einsetzte und die' Dióskuren waren die vorsteher der olympisclien spiete; "Herakles hatte es so verordnet (Pindar. "Olymp. III, 36 sq.). Man wird also auf die sehr alte féer der ofympischen spiele das augenmerk richten müssen als auf den wahrscheinlichen entstehungsgrund des glaubens an epiphanien der Dioskuren während oder nahie der olympischen festzeit beim höchsten sonnenstande. Im bereiche der olympischen spiele selbst mag es manche sage gege. ben haben, yon den friedlichen siegen zu Olympia die unter dem scliutze derastoren gewonnen und durch sie überraschend schnell nach hause gemeldet wären z. b. nach Agrigent an 


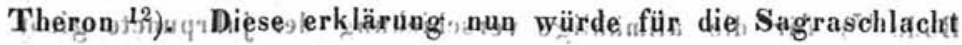

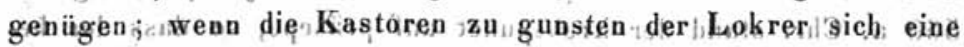
abwesenheit nach-Jtalien gestatteten $y_{0} s p$ mussten sie als treue festwarte rasch wieden hinüber / nach olympia sund $k$ onnten jetzt deńs sieg melden $_{1}$ Atier die epiphanie nach dem siege ${ }_{9}$ des Ae milius führt doch unabhängig auf das solstiz selbst, ader voll.

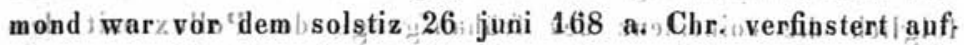
gegangen, dié lolympische feier kam also auf den nächsten avoll-

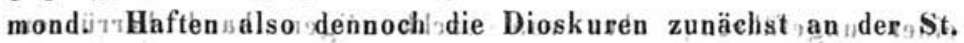
Johannisnacht und einer etwaĩ caufi sie bezüglichen erscheinung am firmament? so idass vein jedes' fest, isei es ein römisches oder

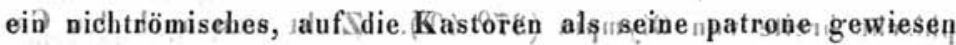

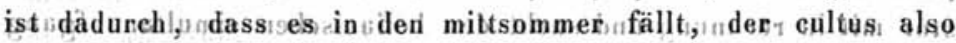
hierınur dası secundäre, die exacterobservation das primäre ele-

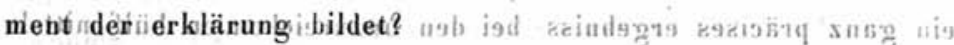

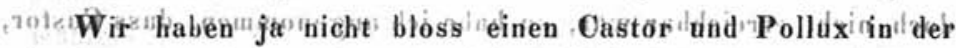
mythologie, sobidern auch an firmament Nun nehme tman eine

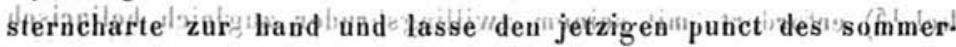

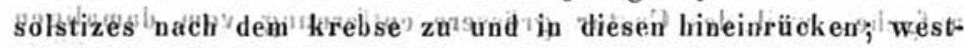

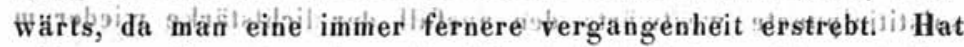
malh' den solstitialpunet ưngefäliribis D im krebs 'geführt' 'so ist ess genug, denti jetzt ${ }^{3}$ steht Pollux etwa $11^{0}$ östhich "davon. ${ }^{3}$ So lange die sonne aúl ihrer jährlichen bahndem Pollux näherats 119

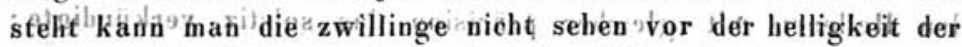
sobne" "aber" "wehn "sie "sich bis $11^{0}$ entfernt hat iso zeigen sie

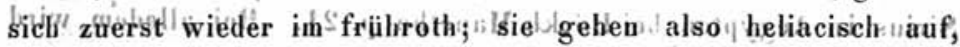
wenn die sonne bei $D$ im krebs steht. Hier aber war das sol. stiz zu einer zeit welche der unsrigen fern liegt und bis in diése vergangenkeit hatten" wir ded solstitialpunct hinaufgeschoben.s Es,war aber, dies die blüliendste zeit yon Hellas, wie man

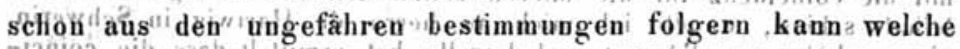

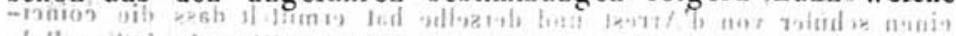

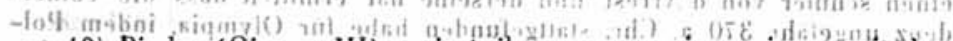
wodu 12) Pindar (Olymp. III), preisat ihn wegen seines sieges mit dem

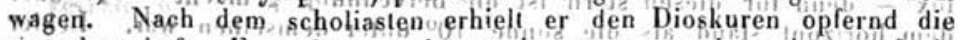
siegesbotschaft. $\mathrm{Er}$ opferle, gevis $\beta$, an dem tage wo sewe schonen pferde auf der hahn, zu Olympia "fuhren und bat dabei die zwillinge der Leda, dass sie, ihm dem, wagen zuerst, ans ziel, zum siege lenkten. Vernielt

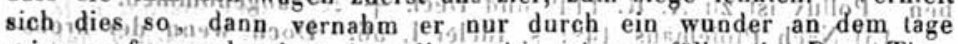
seines lopfers und, gebels dass dies gebet schon erfullt sei. Denn Theron opferte if Agrigent, en war $z H_{1}$ hause geblieben. Sei dem in dess wie ihm, wolle, es ist, an sich selbst, wahrscheinlich dass jenen botendiensten nach schlachten als muster friedlichere zu grunde liegen. 
Bode 23 ) für die allmählige verschiebung der jahrpuncte giebt. 560 a. Chri 1 stand der krebsputict auf präsepe; so dass der heliacische aufgang des Pollux ein par tage vor dem solstiz statt fand, während er elenfalls um ein par tage nach dem. selben einfrat um 63 p. Chr w weil der krebspnnct inzwischen sich zwischen zwilling und krebs iverschoben hatte 1 in folglich die coincidenz đés frühaúfgangs des Pollux mit bem sommersolstiz in die beste zeit griechenlands/fallen und zwar eińer urgefähren, abev von sachkundiger hand herrühren: den rechnung zufolge a. Chru 370 und von Ida aufwärts also etwa bis in die perserzeiten ${ }^{14}$ ) und zwar dies für die geographische breite von Olympia $\left(370^{\circ} 40^{\prime}\right)$. Zieht man auch den Ca. stor mit in rechnung und sucht den heliacischen simultanaufgang beider, ko kan dies allerdings das resultat ändern, hallein da ein ganz präcises ergebniss bei deu unżureichenden hülfsmitteln

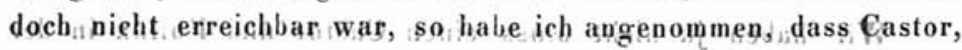
der seiner geringeren helligkeit wegen cinen grosseren sehwiukel ${ }^{15}$ ) erfordert, mit seinem zwillingsbruder zugleich heliacisch aufgehe, weil des Castor grössere entfernung vom damaligen solstitialpuncte westwärts den ausfall der lichtstärke wiederum aufhebe, Mlan wird hierbei nicht übersehn, dass für verschiedene geographische lngen die $_{\text {tage }} \mid$ des $_{\mid}$betreffenden, phänomens sich etwas modificiren, lalsn das gestirn der Dioskuren nicht al.

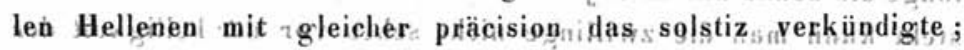
man vergleiche die ähnliehe I frage nach dem $_{11}$ frühaufgange des Sirius in Aegýpten beiBögkh Manethol p 21; ${ }_{2}$ Bei alledem wird

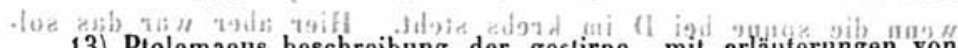

13) Ptolemaeus beschreibung der gestirne, mit erläuterungen von Bode. Berl. 1795 p.246. 192017 w -vilo 14) Da ich mich aus Bodes datirungen der heliacischen auf- und untergänge fur $63 \mathrm{p}$. Chr. r. (a. o. p. 259 ) nicht vernehmen kơnte, während mir" die coincidenz" Pur die alferen zeiten Griechentarids ganz: bnzweifelhaft schien, iwandte inb mich an, herro idr. Hartwig, in Schwgrin, einen schüler von d'Arrest und derselbe hat ermittelt dass die coincidenz ungelahr 370 a. Chr. staltgefunden babe für Olympia, indem Pollux denn für diesen stern ist die berechnung gemacht 1 i über dem horizont stand als die sonne $10^{\circ}$ unter demselben war. Leider standen dem treffichen kenner dieser sachen nicht die erforderlichen hilfsmitel zu gebote um dem resultat die'gewünsché pràcision zu geben Pollux isi zu A. R. $114^{\circ}$ und Decl. $+28^{\circ}$ angenommen. (5) Nach Ptolemăus 14\%: s. Ideler I, 56. Doch wenn der krebspunct $11^{0}$ östlich ron Pollux steht so ist Castor allerdings wohl noch nicht volle $14^{\circ}$ sondern nur efwas über $13^{\circ}$ davon entfernt Hiernach würde fur den simultanaufgang ein noch ălteres solstiz zu'suchen sein als 370 a. Chr.

Brought to you by | New York University 


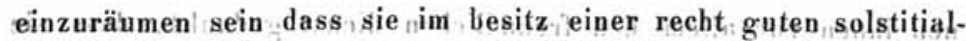
bestimmung waren / für lange zeit, wenn (sie sich an das heliacische/ wiedererschieinen von Castor und Pollux hielten. Bedenke man dabei nur dass noch Meton das solstiz um mehr als einep tag. falschlangegètıen hat ${ }^{16}$ ), er als astronom, währęnd der fest. kalender sich schon elier mit einer ungefähren, conyentionellen satzung begnügen konute, um den heliacischen ${ }_{0}$ Dioskurenaufganig als termin 'zu benutzen; yor welchem die Olympien nicht gefeiert werden sollten. Dies letztere, ist auch zutreffend für die ältesten zeiten, da die beiden , himmlișchen festwarte dann sich früher wiederzeigen $\backslash$ als das solstiz.

Dasdie Olympien zugleich vou der sonne und dem $_{13}$ monde, von jener gleichsam als der stundenzeigerin, von diesem als dem minutenzeiger, orientirt wurden, so bemerkt man wohin die anknüpfung des sonnenstandes" an die matinale wiederkehr

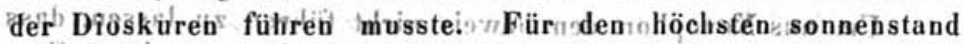

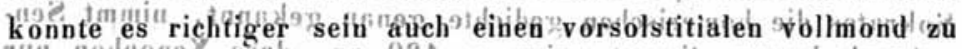
wäblen, wenn er dem mittsommer nâtier tag als der nachfolgende. Wablefn, wenner dem mittsommer naher lag als der nachfolgende.

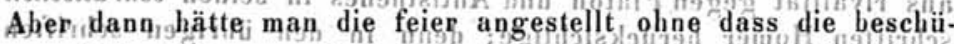
tzergdièseri feier am firmamente gesehen is worden wären, in , den

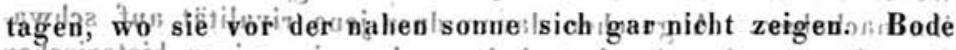
rechnet die zeit "der unstchtbarkelit des Castor "für Róm "aúf ZWölf, für Alexandrien auf sechszehn tage (a. o. p. 259). toin Aus den sagen lässt sich hiernach auch manches deuten. Der zum kampf nausräckende soldat freut sich beim anblicke dêr huldrèiclien Kastoren in der morgenfrülie; aber sie ver. schwinden sofort mit anbrechendem tage. Doch sie sind nur yom himmel verschwunden, um desto thätiger auf erden den schaaren voranzureiten. - Auch ein bote kommt und meldet was zumelden ist; dann geht er seiner wege. - So ist auch jeder heliacísch aufgehendel stern ein zwitter von dunkelound ficht (vgl. Max Duncker gesch. d. alt. III, p. 37) und wiewohl die Dioskuren an sich nichts mit dem morgen und den ersten sonnenstrahlen Igemein haben, wenn sie z.b. den schiffer im sturme retten, die wetternacht durehbréchenid: so haben doch die Helle-

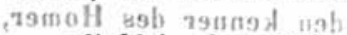

16) Ideler I, p. 326. Sollte Meton vielleicht den morgen gewählt haben mit rücksicht auf eineo heliacischen aufgang? etwa der $z$ willinge? Man musste also den heliacischen aufgang von Castor und Pollux mit der grössten sorgfalt für Athen und 432 a. Chr. berechnen. Unsere hülfsmittel baben leider keine hinreichende genauigkeit gestattet. 
nen ihnen "vor allem den freundlichen dienst gedankt, w dass sie den festmorgen von olympia und den ersebnten / gottesfrieden meldetèn. HU $U_{m}$ die Olympien zu begehen müssen die Tyndariden da sein, d. h. es muss mittsommer"sein; aber auch ihre schwëster Heléna müs die schöne wimper voll aufschlagen, es "muss' zugleich vollmond "sein." Und" sind jene bei neumond noch unsichtbar, "so"mochte man" sagen', dass'sie ihre abhanden gekommene "schwester"suchten (M. Duncker l. c.). Was ein dichter personificirend sei es im scherźl oder im ernste /gesungen, nähm 'man'ganz eigentlich, dem/gottesbedürftigen gemüthe wurde alles und jedes zu persöntichen figewalten

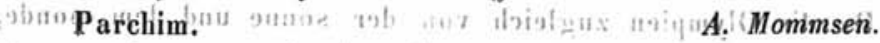

alis moesile tsor

it unstoively

$1+110 j+110 \%$

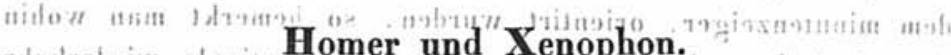

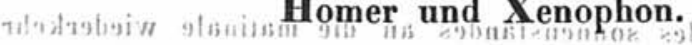

buite Um "aus Xenophon den béweis nicht führen zp, lassen Sokrates die homerischen gedichte ${ }_{i}$ genan gekannt, nimmt Sengebusch homer. dissert. prior. P. 139 an, dass Xenophon nur aus rivalität gegen Platon und Antisthenes in seinen sokratischien schriften "Homer berücksichtige; denn in den" ülirigen "schriftén werde er"selbst"nicht"einmal in den stellen genant; in denen er ihn Inachahme. Abgesehen davon dass jene rivalität auf schwachen ,füsssen ruht, ist festzuhalten, dass in seinen historischen und antiquarischen schriften Xenophon da, wo er den ton aus Homer nimmt, aus gar begreiflichen gründen den dichter vicht nennen konnte, und dass die homerischen nachahmungen ungleich hăufigè in 'Xenophon's' historischen 'schiriften isind, als Sengebusch angiebt: sehr schönè bemerkungen finden, sich darüber bei Heiland Oraestionum \de dialecto Xenophontea capita selecta. Halberst. 1844 , p. 12 sqq. Nur eine stelle will ich hier nachtragen. Xenoph. Cyrop. Vl, 1, 3 heiss't es von Panthea: " $\pi \alpha$ -

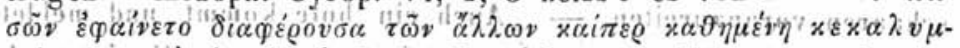

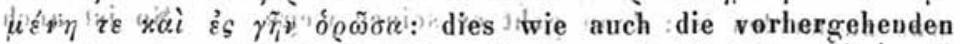
bemerkungen sind durch Helena in Hom. II. $\Gamma, 141$; ajzíkq $\delta^{\prime}$

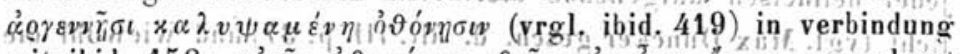

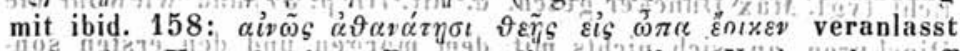
und geben Xenophon's auffassung dieser stelle. Noch mehr stoff giebt es" für dergleichen: "daher" werden wir, zumal da bei den uns lgenauer bekanten sokratikern ntiefes studium dés Homer sich findet, sagen, das durch Sokrates, den kenner des Homer, Xenophou diesen dichter lieb gewonnén habe.

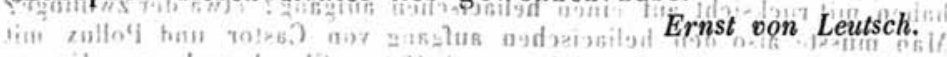

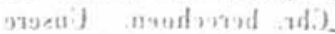

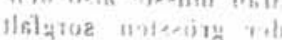

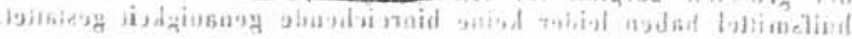

